\title{
Transforming the Multifluid PPM Algorithm to Run on GPUs
}

\author{
Pei-Hung Lin* \\ Lawrence Livermore National Laboratory, 7000 East Ave., Livermore CA 94550 \\ Paul R. Woodward \\ University of Minnesota, 117 Pleasant St. S. E., Minneapolis, MN 55455
}

\begin{abstract}
In the past several years, there has been much success in adapting numerical algorithms involving linear algebra and pair-wise N-body force calculations to run well on GPUs. These numerical algorithms share the feature that high computational intensity can be achieved while holding only small amounts of data in on-chip storage. In previous work, we combined a briquette data structure and a heavily pipelined CFD processing of these data briquettes in sequence that results in a very small on-chip data workspace and high performance for our multifluid PPM gas dynamics algorithm on CPUs with standard sized caches. The on-chip data workspace produced in that earlier work is not small enough to meet the requirements of today's GPUs, which demand that no more than $32 \mathrm{~KB}$ of on-chip data be associated with a single thread of control (a warp). Here we report a variant of our earlier technique that allows a user-controllable trade-off between workspace size and redundant computation that can be a win on GPUs. We use our multifluid PPM gas dynamics algorithm to illustrate this technique. Performance results for this algorithm in 32-bit precision on a recently introduced dual-chip GPU, the Nvidia K80, are 1.5 times that on a similarly recent dual CPU node using two 16-core Intel Haswell chips. The redundant computation that allows the on-chip data context for each thread of control to be less
\end{abstract}

\footnotetext{
${ }^{*}$ Corresponding author at: Lawrence Livermore National Laboratory, 7000 East Ave., Livermore CA 94550

Email addresses: lin32@llnl.gov (Pei-Hung Lin), paul@lcse.umn.edu (Paul R. Woodward)
}

Preprint submitted to Journal of Parallel and Distributed Computing November 20, 2015 
than $32 \mathrm{~KB}$ is roughly $9 \%$ of the total. We have built an automatic translator from a Fortran expression to CUDA to ease the programming burden that is involved in applying our technique.

Keywords: Precompilation, code transformation, GPU computation, computational fluid dynamics

\section{Introduction}

GPU accelerators have been incorporated in nodes of several very large machines over the last few years. Also, recent procurement decisions will put future machines in place with variations on this basic machine architecture. We therefore conclude that scientists wishing to perform simulations for their research using computational fluid dynamics (CFD) techniques must find ways to enable their numerical algorithms and codes to adapt to the special requirements that GPUs demand for good performance. Work by others in this area has been performed especially in the meteorological domain [1, 2, 3, 4, notably by Mikalakes and collaborators at NCAR and more recently by the group at the Swiss national computing center [5]. Success has also been reported by a Stanford group working with a Sandia National Laboratory combustion code [6]. All these other efforts attempt to achieve good performance on GPUs by developing and applying an approach of code modification that could be applied more generally than in one specific context.

In our earlier work with Jayaraj and Yew [7, 8, 9], we have developed an approach of automated code translation, embodied in the CFDbuilder tool [9] and distributed along with the mPPM mini-app available on request via the Mantevo project Web site [10]. This earlier work, stemming from our experience with the IBM Cell processor and the LANL Roadrunner machine [11, especially targets processors whose principal means of delivering high performance is a SIMD engine connected to an on-chip cache or local store. This approach has achieved good performance, even at extreme scale, on multiple large machines [12.

In the present work, we describe a relatively simple way in which the approach of our CFDbuilder tool can be extended through the use of a few additional directive annotations of a code, to allow a programmer to specify desired trade-offs between redundant computation and on-chip workspace size. A single annotated source code can then be transformed in the manner 
of the present CFDbuilder tool for good performance on a cache-based device, using minimal redundant computation, and alternatively for a GPU by specifying the necessary amount of redundant work. A new code translation tool with the above extended capabilities is under construction. In this paper we explain how the trade-off between redundant work and on-chip workspace size is exposed, and we give initial results for our multifluid PPM code to demonstrate the effectiveness of this approach and quantify its benefits.

We note particularly that our translation into CUDA for the GPU adopts a processing model more akin to our earlier work than to the mode of computation for which the GPU device appears to have been designed. The device seems designed to support a sequence of computational kernels, each performed over the entire computational domain. Each kernel is intended to read in data from device memory, modify it to produce output data, and write that data back to the off-chip device memory. For CFD, which does not naturally decompose into kernels, such an approach would involve massive data transfers back and forth between the off-chip and on-chip memories (cf. Table I in [10]). This style of programming puts stress on the weakest part of the computing system, the memory bandwidth to the chip, which is also the largest consumer of electrical power. Instead we design a single "kernel," in the GPU programming sense, which implements our entire grid cell update for a single 1-D pass. Multiple data read and write-back events occur inside this kernel, which maintains a persistent store of intermediate results.

\section{Production Line Analogy}

We will view the update of two adjacent $4 \times 4$ planes of 16 grid cells by analogy to an industrial process taking place on a production line with a conveyor belt carrying partial results from one work station to the next (see illustration in Fig. 1). The items at each work station consist of a collection of 32-word vectors. Some of these arrive from the workstation just up the line on each "chime" of a clock, and some are passed on to the next work station down the line on each chime. Other vectors are generated at this work station, used there, and discarded on each chime. Finally, there is a set of vectors that are made available for use at all workstations (shown in the cloud in Fig. 1). On each chime, these shared vectors are all "barrel shifted" by one unit. This barrel shift can be viewed as moving a series of consecutively generated vectors all down the production line by one station, discarding the vectors at the downstream end, and picking up new vectors at the upstream end. 


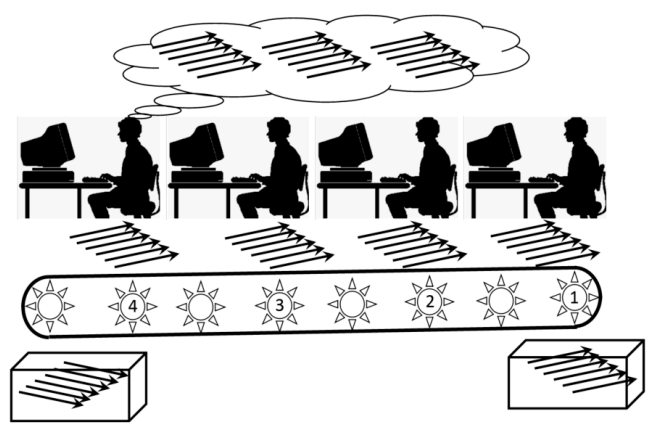

Figure 1: Production line analogy for the operation of pipelined code for the updating of physical state vectors packed into grid briquette records in off-chip memory.

Although we can associate each component vector in such a barrel-shifted series as living at a single work station on the line, they are all available for use at any station. On each chime of the clock, a fresh set of input vectors is provided at the top of the line. These represent the present physical state of 2 grid planes of cells, that is, 32 cells. On this same chime, a set of new, fully updated vectors representing the new physical state variables in 2 grid planes, hence 32 cells, is produced, placed into a new, possibly transposed grid briquette record, and written into a persistent store. The updated grid planes are not the same ones as are now entering this production line; they entered several chimes ago. There is a delay in producing the updated grid planes that can be considered as a pipeline latency for this industrial process.

For those familiar with computational fluid dynamics, we can make our industrial analogy more specific by briefly summarizing what is produced at each station, and at what cost. At work station 1, we produce the densities of individual fluids in the cells and the sound speeds, as well as interpolation parabolae for density, pressure, and x-velocity. This costs us 413 flops/cell, 70.5 of which are redundant. At work station 2, we produce interpolation parabolae for $y$ - and z-velocity as well as the time-averaged pressure and $\mathrm{x}$-velocity at the cell edges. This costs us 219 flops/cell, 33 of which are redundant. At work station 3, we produce the time-averaged fractional volume of a tracked fluid at the cell edges as well as 5 updated moments of the fractional volume of this fluid in the cells. We also produce the fluxes at the cell edges of the mass and x-momentum. All this costs us 231 flops/cell, none of which are redundant. At work station 4, we produce the remaining 
4 updated moments of the fractional volume of the tracked fluid, the fluxes of y- and z-momentum and energy, and finally the fully updated fluid states in the cells. This costs us 233 flops/cell, none of which are redundant. In the cloud in Fig. 1, we have a persistent, constantly barrel shifted set of 96 vectors. At most 232 vectors are live at any point in this process. The production line delay is 6 grid plane pairs.

We can set up such a production line to update our planes of grid cells for either of two reasons, or both. The first reason to do this is to avoid the need to have a copy of each temporary vector associated with the update of a grid plane pair for all of the grid plane pairs in the problem. For our PPM code, these temporary vectors number in the hundreds (232 in fact), while the vectors needed to designate the physical state of the grid planes amount to only 16. Having a production line thus saves us an immense amount of data storage. We need less than a handful of copies of these temporary vectors to get the job done, because we continually reuse them by assigning new values to them upon each barrel shift with each chime of our clock.

A second reason, more familiar to computer scientists, for setting up a production line is to get all the different workers at their separate work stations to work simultaneously, thus increasing productivity. Doing that requires a balancing of the amount of work at each station and facilitating access by all the workers to the shared set of barrel-shifted vectors they all must use. Copies of these vectors may be required, which would increase the clutter on the line. In Fig. 1, we show 4 workers at 4 successive work stations, but it is possible for a single worker to perform the work at each successive station, and then to begin again at the first station at the next clock chime. Such a single worker would never be held up waiting on the output of a colleague. Increasing productivity by getting many workers to do their jobs simultaneously, without one waiting upon the other, is most easily accomplished by setting up parallel production lines and assigning one worker to perform all the tasks at each line. As long as we can afford all the infrastructure that this entails, this is by far the easiest way to extract the maximum work from each worker.

We can think about CPU and GPU processing in a useful way in these terms. Each worker can be viewed as a thread of control. This is the smallest entity in a computer that can perform any task. The sequence of tasks to be performed on a single production line can be thought of as our program. For both the CPU and the GPU, each operation in the program is performed simultaneously and identically on 32 operands, since the fundamental mode of 
execution for either device is SIMD processing. Whether processing these 32 operands takes one simultaneous operation, two, or four is a detail. Logically, the effect is the same as performing these operations all at once. For example, a SIMD processing width that is half as large can be compensated for by having a two times faster clock. As a practical matter, we can think of all our operations on the pipeline as being performed by a SIMD engine. Each worker needs this same tool to do all its work. A fundamental difference between the GPU and the CPU is in the number of such tools that are available, even when clock differences are accounted for. Each processing core capable of executing instructions, even if these instructions are chosen alternately from different threads, has a limited number of SIMD engine tools available. On the CPU, such as the Intel Haswell, there are 16 such cores and each has two 16-wide SIMD engines, which might or might not be operating simultaneously. On the GPU, such as the Nvidia K80, there are 14 such cores, called SMX units, and each has six 32-wide SIMD engines, operating at about $1 / 3$ the clock rate of the Haswell engines. Effectively, this is somewhere between 2 and 4 times the production potential, if all this hardware can be continually fed.

Both the CPU and the GPU use simultaneous multithreading (SMT) to increase production. The core choses instructions to execute from multiple instruction streams, each being what we are calling a thread of control. These threads, as we use the term in this article, are capable of executing completely different sequences of instructions. In our industrial analogy, our threads are the workers. We can assign each worker to do all the tasks of a production line, or we can assign them to do only the tasks at specific work stations. If we make the latter choice, some of the workers may end up being idle as they wait for vectors to be produced by earlier production line stages; if we make the former choice, we must set up an entire production line for each of our workers. This will, as it were, require a much larger factory building. The CPU and GPU are likely to be about the same size and contain about the same number of transistors. In our industrial analogy, this means that they each are constrained to a factory of about the same size. The CPU includes considerable on-chip cache storage capacity, which in our analogy allows the construction of multiple, very long production lines with the implied amount of shared vector storage between the work stations (indicated by the vectors in the cloud in Fig. 11). However, the CPU has a smaller set of SIMD engine tools for use on each such line. The GPU has dramatically less storage capacity for vectors, but it has significantly more SIMD engines in its factory. 
It can then run many production lines, but these must be short. To produce the same final products, our updated physical state vectors, the short GPU production lines must run through the data several times, performing only part of the production job on each run through.

Which design choice is preferred depends upon the nature of the algorithm, or our production method. If the algorithm involves a very large number of intermediate results held in temporary vectors, then with short production lines we will be forced to either write these to off-chip memory only to read them right back in again a short while later, or we will need to rebuild them upon multiple traversals of our short pipelines. In the first case, our workers can be idled waiting for the necessary vectors to be read in, while in the second case our workers might perform a great deal of work that could be avoided by redesigning the factory to allow longer production lines. The CPU choice can also result in waste. It is possible that the large on-chip storage capacity is un-necessary for a particular algorithm. In this case, the CPU's limited SIMD engine count will result in reduced production. It is our task in this work to find a way to express our programs so that after automated transformation by a pre-compilation tool we can have implementations that work well on either type of device. Essentially, this means that we must find a way to write both types of programs, CPU-friendly and GPUfriendly ones, and in addition to that find a way to automatically create both from a single source code expression. We will need to express the arithmetic that must be done in a way that is device-neutral. Then we must design a code translator that performs the necessary adaptations of this algorithm to the peculiarities of either device. This of course is the original concept of a compiler. In our case it will be a pre-compiler. The compiler for each device expects code to be written in a particular style that it is designed to optimize. Our precompiler will need to produce what each device compiler is set up to handle.

In the following sections, we will first explain how we build CPU-friendly implementations for a simple code expression. These will involve very long production lines in which the number of operations performed by the SIMD engines are minimized, since on the CPU the SIMD engines are a precious and limited resource. We will then explain how this implementation can be modified using a key and quite general technique to reduce the need for on-chip storage capacity by performing redundant work. This new implementation will minimize the on-chip storage requirement while allowing the SIMD engine work to expand, since on the GPU storage is precious and SIMD 
engine work is not. We will show how these two implementations can be automatically derived from the same, simple source code expression. We will find that in comparison to other published work our two alternative implementations perform quite well. However, both leave a good deal of room for improvement. We will finally identify a general technique by which we could, from our same source expression, automatically enable more work on each production line to be performed simultaneously. This can be done at a cost of increasing the need for on-chip temporary data storage. The CPU has the storage capacity to support this technique, but there is a "register pressure" price to pay as well. On the GPU, the extra data storage would draw on a precious resource, but the ability to exploit the resulting parallelism may be more readily available. Applying this new technique for exposing instruction level parallelism (ILP) and reporting the results is left to a future article.

\section{CFDbuilder Approach}

CFDbuilder [9] transforms a code written in a simple Fortran-77 restricted style that we call Fortran-W, or Wilhelmson Fortran, into a Fortran expression that standard compilers can turn into very high performance executable code. This automated code transformation applies only to the principal computational section of a code. It is based on just a few key concepts. The first of these is a data structure in which contiguous records in off-chip memory describe individual grid briquettes. Our grid briquettes each contain $4^{3}$ cells. The grid briquette records in off-chip memory are represented in Fig. 1 by the boxes of vectors at the bottom of the diagram. Following the factory analogy, these boxes arrive at the factory door through an expensive process of transport. They must be packaged (because of cache line structure in main memory), and their contents must be minimized in consideration of the expense of delivery. Newly produced boxes of vectors may need to be transposed before packaging, as is indicated in Fig. 1.

In the Fortran-W coding style, we express the arithmetic needed to update just a single briquette by a sequence of nested loops. These may be divided among any number of subroutines. The fast-running index, the first in Fortran, runs over the 16 cells in a grid plane. This produces perfect alignment of all 16-word vectors for SIMD engine processing. For SIMD engines that process 32 words at a time, as in GPUs, we will need this fast-running index to go over 2 adjacent grid planes. This will be an adaptation we will need to make in a GPU-friendly code transformation. We will find that op- 
erating on 2 grid planes of 16 cells will require less on-chip data storage than operating upon single grid planes of 32 cells each. There will be an associated cost in vector realignments, but the GPU has processing power to spare but no data storage to spare. In contrast, this adaptation to the GPU slows code down on a CPU by roughly $25 \%$. We therefore need a code translator that creates 16- or 32-word vector arithmetic depending upon the desired target hardware.

CFDbuilder transforms the Fortran-W code expression by analzing the extents of the outer loop index in the loop nests. It does no dependency analysis, and it assumes the programmer has created the sequence of loop nests so that no such analysis is necessary. (We view the job of CFDbuilder as transforming the program, not debugging it.) CFDbuilder demands that the programmer identify vectorizable inner loops with the now-standard directive "!DEC\$ VECTOR ALWAYS," with, if necessary, a "cDIR\$ IVDEP" thrown in as well. This is no trouble for the programmer, since the program is being explicitly written for a SIMD engine (or else it would not be fed to CFDbuilder) and after 40 years of vector computing it is reasonable to assume that "everybody knows" what is and is not vectorizable.

The grid briquette to be updated is augmented on the left and right sides (in the $\mathrm{X}$ dimension) with an algorithm dependent number of additional ghost grid planes. There is no augmentation in $\mathrm{Y}$ or $\mathrm{Z}$, because these would lead to unaligned vector use. To access data not in the briquette or its ghost regions, the program must read in additional briquettes offset in these dimensions and construct from them properly aligned grid planes (vectors) of offset data. This exposes the burden that the processor actually must bear in doing this. The inconvenience of doing this suggests that it should be very strongly avoided. The requirement that adjacent briquette records be read in underscores the cost of such offset vector construction, and it makes clear that the briquette record is treated as a data atom. One reads or writes an entire briquette, not just a portion of one.

It will generally be the case that in each subroutine loop nests with the greatest extents in the outer loop index come first, because succeeding loop nests with lesser extents will use the products of these earlier loops for multiple values of the outer index. CFDbuilder does no analysis to verify this, because none is necessary. This structure is demanded by code correctness. It results in CFD applications from simple causality - information is transmitted by waves propagating in the $\mathrm{X}$ dimension in an X-sweep of the algorithm. This loop structure creates a natural opportunity for code pipelining. If two 
loops are fused, with the first providing two planes of data that are both used in the second, then a large amount of temporary information need not be stored. This can be seen in the very simplified code below:

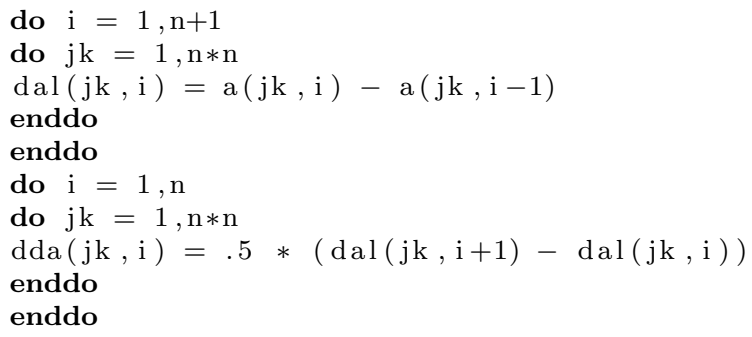

As written, in Fortran-W style, these 2 loop nests require temporary storage of an entire 2-D array dal. CFDbuilder will fuse them and remove the unnecessary storage, producing:

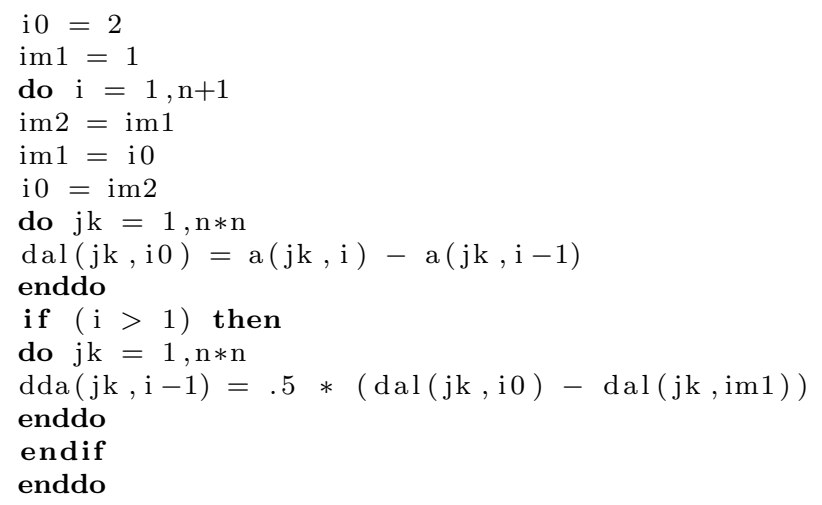

For such a small calculation, this transformation may seem hardly worth the trouble, but in CFD programs the loop bodies involved can run to 100 statements or more, and the storage saved can be enormous. The pipelined code also executes faster, because the tiny 2-plane vector dal will never leave the closest cache. CFDbuilder carries out this transformation extensively, in response to the programmer's directive "cPPM\$ longitudinal loop," which must be placed in front of each loop to be fused. A subroutine in-line directive "cPPM $\$$ inline" forces CFDbuilder to inline any subroutine of any length, and enables CFDbuilder then to fuse all the loops inside it with loops originally inside many other subroutines. In this way, the code expression for our multifluid PPM gas dynamics algorithm is turned into a single outer loop over grid planes that contains many inner loops over 16 grid plane elements and that overall involves more than 1000 vector flops per grid cell. Over many years of experimentation, we have found this to be the highest 
performing PPM code expression on all CPUs we have worked with since 2003. It can sometimes be speeded up still further by adding in front of each vector loop the assertion "!DEC VECTOR ALIGNED." The alignment should have been obvious to the Fortran compiler, but it cannot hurt to add this information, just in case the compiler had not noticed. With our multifluid PPM algorithm we are using in this study, CFDbuilder generates a transformed code expression that achieves $18.4 \%$ of the peak performance of the Intel Sandy Bridge CPU (see Table 1).

On the CPU, it is possible to fetch an entire grid briquette record all at once without causing the code to stall. Consequently, CFDbuilder generates briquette record prefetching one entire briquette processing interval in advance. This makes for a relatively simple code expression. The programmer can assume that all the contents of the briquette record are available anywhere in the giant fused loop (their general availability means they reside in the cloud in Fig. 1). This is a luxury afforded by the CPU's relatively spacious on-chip cache memory and by its ability to overlap with computation quite a large number of cache line fetches. Present GPUs cannot do this, although GPU designs are changing in this respect in very positive ways. We will see below that in our GPU code transformation we must accommodate a far more limited ability of GPU cores to prefetch data and, of course, to find a place to put it on the chip until we can make use of it.

One final aspect of the CPU code translation is notable. Our code performs 1-D passes through our data, in a sequence XYZZYX. The grid briquette records can be fetched onto the chip equally efficiently regardless of the order of these fetches. However, the contents of the records must be transposed for SIMD processing. The transposition is performed on chip, when it should take very little time. Nevertheless, neither the CPU nor the GPU hardware is very good at this operation. It must be expressed with care in the Fortran-W source, with, we find, the transposition of 4 variables at a time (and definitely not more than 4) contained in single loop nests. The Fortran-W is written so that in either sequence of passes, XYZ or ZYX, a temporary grid block array is used in 2 of them. This temporary array can be sized to fit into an L3 on-chip cache for a CPU, which will eliminate $2 / 3$ of the off-chip data transfers in the algorithm. On present devices, this technique is overkill. Nevertheless it draws attention to an advantage of the CPU for much more extensive data reuse than the present GPU, which has only a tiny on-chip shared cache. As chips continue to increase in processing speed, techniques like this could become essential programming tools. 


\section{CFDbuilder Modification}

\subsection{Asynchronous Fetching of Off-Chip Data}

As explained above, the GPU's limited on-chip data storage capacity forces us to bring onto the chip the absolute minimum data we need for our grid plane update. Its SIMD processing width also forces us to adopt 32-word vectors. To adapt our CFDbuilder approach described above, we then have 2 choices. First, we could bring in 2 briquette records and update single planes of 84 cells formed by taking a plane from each briquette and placing them side by side in 32 -word vectors. This choice would double our on-chip data storage requirement. For a single thread of control, the GPU does not allow enough data to be held on the chip to enable this approach, Our second choice is to place two grid planes from the same briquette side by side in our vectors and work with those. This also increases the storage requirement, since all working vectors are twice as long, but it does not double the requirement. This is consequently our only viable choice. We can avoid bringing entire briquette records onto the chip at once, as we do on the CPU, by rearranging the contents of the briquette record. We do this by storing 32-word vectors for each of the physical state variables (we have 16 of these) together in half-briquette records and fetching these in place of the entire briquette. We must still hold 2 copies of updated briquette records on the chip: one into which we place transposed 32-word contributions as we generate them, and a second that we must leave untouched during the substantial time that it takes to be written back to the off-chip memory, 4 vectors at a time. This on-chip storage cost is 64 vectors of 32 words, or 8 $\mathrm{KB}$. This is in addition to the storage we need for the data we are reading in at the top end of our production line. We note that this $8 \mathrm{~KB}$ is $25 \%$ of all the storage available to one thread of control on the GPU. Happily, we can store half of it in the "shared memory" cache, which provides $6 \mathrm{~KB}$ per thread of control. We are left with just $28 \mathrm{~KB}$ for all our work.

It is not enough on the older GPUs, such as the Nvidia K20, to prefetch half-briquette records. The processor will stall if we ask a thread to fetch more than a single vector at a time. Gratefully, this vector can be designated as type float 4 , so that we can prefetch 4 contiguous vectors without stalling. On the K80 GPU, this constraint has been relaxed. However, if we want our program to run on GPUs manufactured as recently as 2 years ago, and if we do not want the computation to stall on each fetch, we must read in our data in globs of 4 vectors. For our multifluid PPM code, this means we must fetch 
each half-briquette in 4 pieces. This requirement produces a very unusual demand on the program: it must try to consume this in-coming data in globs, which requires a rearrangement of the work of the algorithm. In our implementation we have done this, but were we to rewrite the program, we would not accede to this apparent requirement. It places unreasonable constraints upon the algorithm design. Instead, we would simply change the code to be fetching the next half-briquette rather than the one we are presently updating, which would require an additional $2 \mathrm{~KB}$ of on-chip storage. That would make it possible to overlap each data fetch with any computation that needs to be done, in any convenient order. This freedom would make the program much easier to write, and therefore would be well worth the loss of the 2 $\mathrm{KB}$ of on-chip space. In fact, if we end our program with each grid brick update and begin it again with the next, we could regard the in-coming data as read-only and store it in the texture cache. This would be less computationally efficient, as our present program allows some threads to begin a new grid brick update while others complete the previous one. The slight loss of performance might be worth the $2 \mathrm{~KB}$ of space it would free up.

In summary, the GPU program must orchestrate an elaborate series of prefetches of half-briquette data. The programmer must decide what computations should be performed while these fetches take place and make sure that the incoming data is not used until after the time allotted in the program for it to arrive. In addition, the briquette data structure must be redesigned to accommodate half-briquette fetches, and the pipelining of the program must be significantly altered to enable grid plane pairs, rather than single grid planes, to be the operands of all arithmetic. This last requirement for alternative pipelining strategies can be handled by a code translator and need not be a concern of the programmer. However, the orchestration of the fetches and the fundamental data structures are programmer responsibilities.

\subsection{Episodes of Computation}

The above considerations suggest structuring the program in explicit episodes of computation. These constitute the work that is performed during the time needed for each data transmission. In our implementation of multifluid PPM, we have 4 episodes, and when we must generate data for visualization output, we have 10 episodes. To keep the code execution from stalling, one needs to insert enough work into each episode. How much work is needed depends upon the computation rate that is achieved. For our code, this turned out to be far below the peak rate available in principle, so we 
had no problem at all in identifying enough work to overlap all our off-chip data accesses. This was an unexpected result, and in further work we intend to apply further code trans-formations in the hope of increasing the performance. Nevertheless our achieved performance was quite good.

The episode of computation forms a natural code unit. It begins with a data transmission request and ends with a copy of the acquired data to an on-chip vector. We have chosen to encapsulate each episode in a subroutine. This allows us to set up whatever temporary vectors it requires on the subroutine stack, and to be assured that they will be discarded upon exit. This is very helpful in managing the overall stack of the computation, which is the most difficult aspect, we find, of GPU-friendly programming. To help keep track of the size of the ever-changing stack of working vectors on the chip, we pass into each episode routine the required collection of scratch vectors. Keeping the data workspace small, which is a critical concern on the GPU, is a matter of constantly overwriting and repurposing scratch vectors. This is difficult to do while maintaining a readable code. We find that introducing an unnecessary number of subroutines is a useful technique to make stack management compatible with readable code. Our code translation tool will inline everything, so we do not need to be concerned with the cost of subroutine linkage, nor do we need to concern ourselves with how many arguments each routine is given. This technique produces too many routines, with far too many declaration statements, but it does allow us to have a single vector, over the course of the algorithm, be used in many quite different ways.

This sort of stack management is completely unnecessary on the CPU, because in fact it has a cache, not a user-managed local store. On the CPU, we can simply create as many vectors as we need, and the ones we do not need any longer will automatically be expelled. As long as these expelled vectors fit into the L2 cache, their expulsion costs us nothing. If they must be expelled to off-chip memory, that process will needlessly eat up our memory bandwidth. CFDbuilder therefore does not rely upon this automatic expulsion mechanism. However, it does not go to un-reasonable lengths to minimize the number of these vectors either. This makes CFDbuilder easier to write. For it to manage vectors as required on a GPU, it will need important new features. These are possible, of course, but difficult. In the present work, we have performed the stack management function manually in the Fortran source. One possible technique for future work is to assume that GPU compilers already possess the required stack management features and simply let them do that work on a CUDA translation of a Fortran-W 
program.

\subsection{Processing 2 Grid Planes at Once}

For the GPU, we need CFDbuilder to generate a pipelined code that updates 2 adjacent grid planes at once. This is a straightforward modification of the technique discussed earlier for the single grid plane case. Its only special requirement is that we must repeatedly form offset vectors by putting together the second grid plane of one vector with the first grid plane of another. This is a very simple operation, but it is not fast on either a CPU or a GPU. We find that it requires 4 such vectors to be made simultaneously to run best, and we try to provide this number when possible. An enhanced CFDbuilder tool will be able to handle this consideration automatically, and the programmer can simply ignore this issue.

\section{Results}

\subsection{Performance on CPUs and GPUs}

As a demonstration that the technique of automated code transformation is possible and beneficial, we have applied it to the multifluid PPM algorithm specialized to the case of flow for which the Mach number is less than about 2. In this case, the difference stencil is simplified so that each 1-D pass requires data only from the briquettes of the grid pencil that is updated; no data from adjacent briquettes in the transverse dimensions is required. Calculations of this type with essentially the same algorithm have been reported recently in [14] (see also [13]).

We have manually performed the modifications to our CFD-builder approach that are outlined above and built a new code translator with the ROSE compiler framework [14] that converts that Fortran first into $\mathrm{C}$ and then into CUDA. The performance that results on the Nvidia K20, K40, and K80 GPUs is given in Table 1. Also in the table we compare this performance to that of the code generated by CFDbuilder from a Fortran-W source performing the same arithmetic. Running our manually generated Fortran, with its 32-word vectors, many required vector realignments, and its subroutine call trees managing its stack, produces performance on all the CPUs in the table that is about 25\% slower. This Fortran expression, tailored to the GPU, is therefore not optimal for CPU execution and should not be used to assess the speed with which the CPU can perform this arithmetic. 
The performance numbers in Table 1 are for the update of cubical grid bricks 46 briquettes on a side, hence with $368^{3}$ cells. Such large grid bricks make the cost of starting up our production lines relatively small (about $2 \%$ ) and keep the cost of computation in ghost cell regions that must be duplicated by other nodes in a large MPI run also quite small (about 4\%). Updating smaller bricks is less efficient in terms of these overheads in redundant computation, but it should still proceed at pretty much the above Gflop/s rates on each device.

\begin{tabular}{|l|l|l|l|l|}
\hline Device & GHz & $\begin{array}{l}\text { Threads of } \\
\text { control } / \\
\text { cores }\end{array}$ & $\begin{array}{l}\text { Gflop/s } \\
(32- \\
\text { bit }\end{array}$ & GB/sec \\
\hline $\begin{array}{l}\text { dual AMD } \\
\text { Interlagos } \\
\text { node }\end{array}$ & 2.3 & $32 / 32$ & 71.7 & 8.30 \\
\hline $\begin{array}{l}\text { dual Intel } \\
\text { Sandy } \\
\text { Bridge }\end{array}$ & 2.0 & $32 / 16$ & 94.4 & 10.9 \\
\hline $\begin{array}{l}\text { dual Intel } \\
\text { Haswell } \\
\text { node }\end{array}$ & 2.3 & $64 / 32$ & 301 & 34.8 \\
\hline Nvidia K20 & 0.73 & $168 / 14$ & 121 & 38.9 \\
\hline Nvidia K40 & 0.75 & $180 / 15$ & 136 & 44.5 \\
\hline Nvidia K80 & 0.82 & $416 / 26$ & 432 & 67.4 \\
\hline
\end{tabular}

Table 1: mPPM 1-D Grid Pencil Update Performance

The GPU performance measurements in Table 1 do not include the cost of redundant computation of a different sort. This is redundant work that we do in order to keep the on-chip collection of temporary results small. The full grid cell update is built up from many individual operations, each expressed in a subroutine that might also call additional subroutines. Each of these operations can be pipelined in the manner that CFDbuilder uses and that was illustrated in the simple example in section 3. However, doing that pipelining forces us to save on the stack of the entire algorithm results from each pipeline stage (each loop nest in the Fortran-W program from which multiple results are utilized with different values of the outer array index). For the simple example of section 3, with just 2 pipeline stages, we must save a single grid plane, $\operatorname{dal}(j k, i m 1)$, in addition to the one we generate, $\operatorname{dal}(j k, i 0)$, throughout each iteration of the fused outer loop on i. If, however, we choose to go through two iterations of the first inner loop in 
order to generate only one result, $d d a(j k, i)$, then we would not need to save $d a l(j k, i m l)$ for use when we come back through the outer loop on the next round. This would transform the fused loop to read:

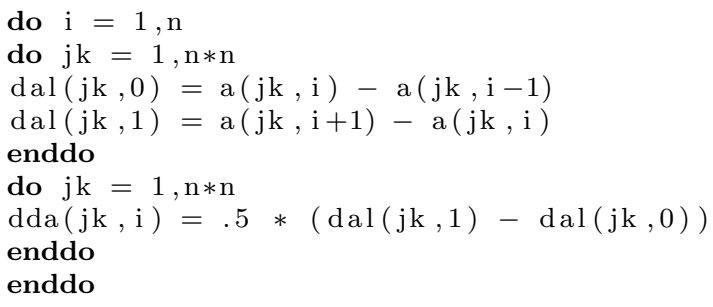

The 2-grid-plane array dal can be discarded or overwritten immediately after this loop nest is executed. In this very simple example, the saving in data storage is small, but when the fused outer loop extends over our whole grid cell update procedure, it can contain thousands of Fortran lines. Then we will not come back to use our saved values in $d a l(j k, i m 1)$ for a very long time. On a GPU, with very little on-chip space, we can be better off doing the extra work in the operations leading up to our desired partial result, $d d a$ in this example, and then having the temporary storage, here represented by dal, available for reuse in the rest of the algorithm.

At this time, we believe this choice of whether to perform the extra work and have the on-chip storage freed or to pipeline the computation to eliminate redundant flops should be left to the programmer. If the programmer expresses a choice through a directive to an enhanced CFDbuilder tool, and then CFDbuilder performs the code transformations implied by it, this choice is not a great burden. Most likely, an afternoon of playing around with such possibilities would be sufficient to get very close to the optimal code for any given hardware. The final choices could then be encoded in C-preprocessor flags that specify the directives to give to CFDbuilder, and these choices could be made contingent on other C-preprocessor flags that specify the computing platform. Without automated code translation, this afternoon of experimentation that we envision here could easily become a couple of months.

In our GPU-friendly, manually generated code expression, we have made a set of choices like those discussed above. In particular, we have demanded that all the redundant flops needed to arrive at a single grid plane pair of interpolation parabolae be performed on each iteration of our fused outer loop. On each such iteration, we generate one fully updated grid plane pair, once the pipeline has been fully primed. We can quantify the savings in 
on-chip data storage from doing this. Because we form such interpolation parabolae for 5 variables, our savings is magnified by that factor of 5 , which makes it nearly 60 vectors. This is no small savings for a device that affords us no more than 256 vectors overall. This savings comes at a cost of about $9 \%$ extra computational labor. On the GPU, this is definitely a win; on the CPU it is definitely not.

We stress that without this technique that allows us to effectively dial adjustments in the trade-off between redundant arithmetic and on-chip data storage, our multifluid PPM algorithm could not, we believe, run on the GPU at speeds competitive with the CPU. This belief is based upon our ability to quantify the off-chip memory bandwidth that would be required to decompose our algorithm into smaller kernels and to run each of them over the full grid of the problem in succession.

\begin{tabular}{|l|l|l|l|l|}
\hline Episode & Vector \\
In/Out & $\begin{array}{l}\text { Vectors } \\
\text { Required } \\
\text { on Chip }\end{array}$ & $\begin{array}{l}\text { Flops per } \\
\text { Vectors } \\
\text { in+out }\end{array}$ & $\begin{array}{l}\text { Flops / } \\
\text { Off-Chip } \\
\text { Vectors } \\
\text { In+Out }\end{array}$ \\
\hline 1 & $34 / 53$ & $68+85$ & $413 / 87$ & $413 / 8$ \\
\hline 2 & $35 / 38$ & $68+90$ & $219 / 73$ & $219 / 8$ \\
\hline 3 & $56 / 40$ & $68+95$ & $231 / 96$ & $231 / 8$ \\
\hline 4 & $73 / 16$ & $68+92$ & $233 / 89$ & $233 / 8$ \\
\hline
\end{tabular}

Table 2: Characterizing mPPM's 4 Episodes of Computation

In Table 2, we see in the second column the number of input vectors and output vectors we have for each of our 4 episodes of computation. In the third column, we also see the number of vectors that must reside on the chip for each episode. There are 68 vectors that all episodes need. These are the 2 copies of an updated briquette record to write back to off-chip memory plus the single glob of 4 vectors that we are fetching from that memory in each episode. Transient vectors that are needed to actually perform the arithmetic are not included in these counts, but the CUDA compiler tells us that it needs 232 vectors to avoid any non-scripted off-chip memory accesses. Thus it seems to be add-ing to our numbers in Table 2, about 70 vectors. We can demand that it add less, but at least on the K80 GPU this results in lower performance. On the K20, performance can be increased slightly by asking the compiler to limit the vector count and then to produce more simultaneous threads of control. However, this increase is quite modest.

Table 2 shows that if we were to build separate programs out of our 4 
episodes and execute them on all the data one after the other, the bandwidth to the GPU chip would need to be increased by about one order of magnitude. In the present implementation, the off-chip memory bandwidth is strongly underutilized, but only by about a factor of 5 , we believe. This indicates that were we to break the calculation up into 4 kernels, the performance would be likely to drop by about a factor of 2 , as it would then become memory bandwidth limited.

\subsection{Probable Causes of Performance Loss}

The performance that we achieve and report in Table 1 is quite good when compared to that achieved normally. For example, the performance of 71.7 , if scaled to 22,000 nodes of the Blue Waters machine, on which we did run a version of our code in 2012, would result in an aggregate performance of 1.57 Pflop/s. On this number of nodes, our code actually ran at $1.25 \mathrm{Pflop} / \mathrm{s}$, but it was executing a more robust version of the algorithm that does not run as fast on a single node as the version we use here, which is appropriate only to flows below Mach 2. At that time, in 2012, only 4 applications achieved over 1.0 Pflop/s performance on Blue Waters, so this CPU performance of our code is quite good. Clearly, the performance on the Nvidia K20 GPU, which is in-stalled on 4000 nodes of the Blue Waters machine, is even better. Nevertheless, we could hope for still better results than we have in Table 1 . The peak performance of the devices involved is much higher, even allowing for the fact that, outside of linear algebra, the multiply-add instruction that is responsible for doubling this peak is not of much use.

In the far-right column of Table 1 , we give the continuous, uni-directional memory bandwidth to the CPU or GPU chips that is required to run at the speeds that are given in the column to the left of this. These bandwidths are not small, but they are not large either. Because all arithmetic in our code implementation is over-lapped with data prefetches and asynchronous write-backs, the continuous bandwidth requirement is a realistic measure of the actual bandwidth the algorithm consumes. All these devices offer much more bandwidth than this, which means that they should be able to run our code much faster if this bandwidth to off-chip memory were the limiting factor. We believe that it in fact does not come close to explaining the delivered performance on our code from these devices. Instead, we believe that it is the ability of these devices to perform computations with on-chip, perfectly aligned vector operands that determines our code's execution speed. 
We might well ask that if the cores are not waiting on the delivery of offchip data, then what is holding them up? A complaint that we have heard from computer vendors arising from our earlier sPPM benchmark code, written in the late 1990s, is that it involves too much "register pressure." This means that we are constantly computing intermediate results that need to be spilled from the registers to the L1 cache in order to make room for more computation. We are not immediately consuming these intermediate results, as we would be if we executed dot-product code, like LinPack. Instead, we want to save the results and reuse them later, but not a great deal later. This is clear from the size of the data contexts for our 4 episodes of computation in the present work. These results are not saved for long, and they fit into the CPU cache, but we do save them for reuse and do not simply recompute them later. With the sPPM benchmark, vendors were invited to insert additional computation, and they were allowed to count it in their performance measures, but only if it resulted in a faster code execution (faster "time to solution"). No vendors did that. Perhaps such a strategy could make our present codes run faster, but we strongly doubt it.

On the CPU, we believe that the limiting factor for our code's performance is the bandwidth on the chip between the L1 cache and the register file. We must spill results in registers in order to make room to compute more results, and we think the bandwidth to do this is insufficient. We come to this belief from observing the performance of our codes over the years on Intel CPUs that have different ratios of this bandwidth to the number of operands needed to produce the peak performance. Our code's performance tracks that ratio quite closely. Based on our discussion of the code's structure, this should not be surprising.

On the GPU, we believe that the limiting factor for our code's performance is the compiler's ability to find instruction level parallelism in the instruction stream executing in each $32 \mathrm{~KB}$ of on-chip local store (the registers of a single warp in our case). This impression comes from viewing the output of Nvidia's performance monitoring tool. The large number of clocks it takes for a flop to be completed once its operands have entered the processing pipeline of an arithmetical unit make it difficult on this hardware to keep the unit busy operating on just a single instruction stream. This pipeline latency is much smaller on the CPU, as far as we know. Nvidia experts suggest that a solution to this problem is to generate more identical copies of the instruction stream, a concept that they call "occupancy." There is no question that this strategy would increase pipeline utilization, but it would come 
at the cost of duplicating our entire on-chip data contexts, which is simply not possible on the GPU hardware. Using our industrial process analogy of Fig. 1, this would correspond to creating entire new production lines to enable the work of each additional worker. The infrastructure required by this strategy, which here takes the form of on-chip data, is immense. The CPU can afford to indulge in this approach, but the GPU cannot, we believe, at least not on our algorithm.

Our performance in Table 1 for the Intel Sandy Bridge node represents $18.4 \%$ of its peak performance. Each core is capable of performing 2 SIMD flops (16 flops) on each clock cycle, while on our code it produces only 0.37 SIMD flops (3 flops) instead. Given the hardware on the core and given the near uselessness of the multiply-add instruction, all we reasonably could hope for from this hardware would be 1 SIMD flop/cycle (8 flops/cycle). Thus we see we are doing fairly well, although one could hope for more. Our performance in Table 1 for the K20 GPU, which exceeds that we achieve on the Sandy Bridge node, represents 11.8 flops/cycle/core, which is 0.37 SIMD flops/core/cycle. This is the same number we achieve on the Sandy Bridge node, where the SIMD processing width is 4 times smaller but the clock is 3.2 times faster. These two devices were introduced around the same time, so it is fair to compare them directly. The resulting performance in SIMD flops delivered per core per cycle is identical, but the K20 GPU is capable in principle of delivering not Sandy Bridge's 2 but instead 12 SIMD flops on each cycle. We therefore achieve a much smaller percentage, just 3.1\%, of its peak performance on our code. We might therefore be motivated to search hard for ways in which our code's GPU performance could be increased still further.

\subsection{Generating Instruction Level Parallelism}

Instruction level parallelism (ILP) refers to a capability to perform multiple instructions taken out of a serially ordered instruction stream simultaneously. This is only possible if there are no data dependencies between them - that is, if the later instruction does not require any results generated in the earlier one in order to execute. Identifying instructions that can be performed in parallel from within single instruction streams (single threads of control) is difficult. It requires extensive analysis, or, equivalently, extensive program knowledge that might be possessed by the program-mer. In the case of our codes, we can identify a way in which large amounts of ILP can be 
produced with essentially no effort. However, this exposure of ILP comes at the cost of consuming more on-chip data storage.

We can explain this technique for exposing ILP best by using our factory analogy in Fig. 1. ILP can be viewed in that context as enabling multiple workers at different stations on the production line to work simultaneously. The production line is an inherently serial process. The work at station 1 must be performed before the work at station 2 can begin. This is the case in the situation where we have a single worker on the line. That worker begins at station 1 , does all the work there, shifts the product vectors down the line one station and then does all the work at station 2, etc. This is simple, the worker is never stalled, and we get all the work out of this worker that is possible. This is all we could ever hope for if all the work on the line must be performed by a single tool, in our case the SIMD engine. However, we have just noted that the SIMD engine tool needs to be fed by multiple independent streams of instructions, which in the factory analogy are multiple objects thrown on each clock into its maw. The work at a single station does not provide the opportunity to keep the SIMD engine properly fed, no matter how industrious our worker is. One simply has to wait for the products to come out of the engine a decent interval after their ingredients have been cast into its maw. With-out these products, the work simply cannot proceed at this station.

To keep the SIMD engine busy, we could toss things into it from the same station on multiple parallel production lines. This approach cannot fail to keep the engine busy. It is also easy to manage, because the instructions being executed are actually identical, which is a great simplification. However, the drawback, as we have pointed out earlier, is that we need to provide all the infrastructure for all these separate, identical production lines. On the GPU, this need to support many parallel threads of control on each core has resulted in the provision of no more than $32 \mathrm{~KB}$ of data workspace to each, a requirement with which we have struggled in devising our coding strategy outlined above. For this reason - the lack of sufficient on-chip data storage - we seek some other means of coming up with independent operations that the SIMD engine can perform, drawing solely on the work of a single production line.

To see how this can be done, we need only visit a real production line, in something like an automobile factory. Work proceeds at each station simultaneously. It is still true that all the work at station 1 must be complete before that at station 2 begins, but the line is very carefully designed so that 
the work at each station takes precisely one chime of a clock. In our case of CFD programs, this condition would be very difficult to achieve, if it is even possible at all. But the work on our production line is repetitive. We can always guarantee that the work at any given station can proceed by delaying it for one whole round - that is, for a single chime of the clock. In our factory analogy, this corresponds to moving the work for station 2 down the line to station 3 , and moving all subsequent stations down one to accommodate this change. This has the effect of lengthening the production line, which has a cost in infrastructure that is required. The second worker enhances the products of station 1 not from one chime ago but from 2. This means that the immediate products of station 1 sit around without continued assembly for a whole chime. We require a place to put them for this short interval, which is more trouble than it might seem. But this trouble can be very small compared to the trouble of finding space for an entire, separate and parallel production line.

Quite generally, if we insert a delay of one outer loop traversal (one "chime") in between each of our work stations (our episodes, for example), then the work at every such station can proceed in parallel without concern for data dependencies. If we insert a one-chime delay before every episode, then every vector that we need to pass from one episode to the next will need to be one grid plane pair longer. Insofar as many of our vectors come at least in pairs (every vector that persists around the entire update cycle, and we have many of these, must come at least in a pair), this lengthening of our vectors will not cost us as much storage space as provisioning a whole new production line. By judicious use of this technique for automatically exposing whole swathes of ILP and combining it with use of our earlier technique of reducing on-chip storage by automatically generated redundant computation, we might be able to find a balance at a performance level significantly higher than is reported in Table 1. Testing this conjecture we leave for our future work.

\section{Related Work}

Our work on the CFDbuilder tool and related code transformation techniques began with our development, with Jagan Jayaraj and William Dai, of a multifluid PPM code for the Los Alamos Roadrunner machine beginning in 2006. This work's extreme pipelining of the briquette record processing uses techniques that are similar to the work for the GPU by Hwu and collaborators 
which they term as "thread coarsening" or "granularity coarsening" [15, 16]. In [16], they also mention data structures which are similar to our briquettes and that, in the GPU community, are referred to as arrays of structures of arrays. The work of Unkule, Shaltz, and Oasem [17] also involves development of automatic tools to handle trade-offs between on-chip data size and redundant work.

The work of John Mikalakes and collaborators with GPU acceleration of the WRF meteorology code is related work within the specific domain of computational fluid dynamics (CFD) [1, 2, 3, 4]. The techniques we discuss in this paper are more difficult to apply to codes like WRF, which use non-directionally split numerical algorithms and data structures that do not exhibit the hierarchical structure of our briquette records for the grid domain updated by a single MPI process. Work by the group at Stanford on Singe [6] has been applied to a combustion code that is more similar to our PPM code than WRF, but this work focuses more on a technique, warp specialization, that addresses similar issues to ours but in a different way.

The group at the Swiss national supercomputer center, CSCS, has carried out a very detailed study to optimize the COSMO regional weather and climate model on GPUs (cf. [5]). This work, like ours here, explores the tradeoff between performance on the GPU and redundant computation. They also have developed a stencil loop language and a code transformation tool, Stella, to assist in generating high performance and performance portable code. The COSMO code is certainly a more difficult target application than our multifluid PPM, but the components of this code are likely to be similar in several ways to ours. Their domain-specific language (DSL) approach is unlike ours in that it goes well beyond annotations of an existing language. Our Fortran input code can be compiled as is, and it will run in that form fast enough to allow debugging and testing before code translation. Stella is a DSL that is embedded in $\mathrm{C}++$ using template meta-programming, while our approach begins with standard Fortran. A report on implementing a convection-diffusion problem in Parareal using Stella is given in [18]. A speedup of this application by a factor of about 4.5 from running on the GPU (a K20) over running on a single Intel Sandy Bridge CPU is quoted. This speedup is similar to ours in Table 2, where we see the K20 performance of mPPM on a K20 GPU to be 3.23 times that of our performance on a single Sandy Bridge CPU. The parallel performance, in Gflop/s, on the Sandy Bridge CPU was not stated in [18], but we suspect it is not as good as our Sandy Bridge performance in Table 2 , because it attains a speedup on the 8 
cores of that CPU of less than 5 times that of a single core, while our code's speedup from 1 to 8 cores on this CPU is a factor of 6 . In [19], performance of $51 \mathrm{Gflop} / \mathrm{s}$ per GPU is reported for the ASUCA weather code running on 4108 Nvidia K20X GPUs. It is likely that this performance figure is significantly less than our 121 Gflop/s in Table 2, because it includes MPI networking costs.

At a talk at the CHANGES 2014 workshop in Beijing, Wen Mei Hwu reported in a single slide on "initial production use results" from 3 applications running on the Blue Waters computing system at NCSA [20]. The presentation slides are short on explanatory detail, but the speedup factors quoted, namely 1.8 (NAMD, molecular dynamics), 2.4 (Chroma, lattice QCD), and 2.7 (QMCPACK, quantum Monte Carlo electronic structure) of a node with a single AMD Interlagos CPU and a single Nvidia Kepler K20 GPU over a node with two of these CPUs only, are consistent with our results in Table 2. We find that the K20 GPU runs our special implementation of our mPPM algorithm 2.4 times faster than 2 of the AMD Interlagos CPUs run that same Fortran, GPU-friendly code on a node of the Blue Waters system. If instead we compare the K20 performance to a Blue Waters node running CFDbuilder's more optimal code for the CPU, this speedup factor of 2.4 drops to 1.7 .

\section{Summary}

We developed a method of extreme pipelining of the processing of grid briquette data structures that results in extremely small on-chip data workspaces for implementing CFD on CPUs. The CFDbuilder tool was developed to automate to computational pipelining and was distributed along with the mPPM mini-app in the Mantevo project. Here we describe a simple way in which the approach of our CFDbuilder tool can be extended through the use of a few additional directive annotations of a code, to allow a programmer to specify desired trade-offs between redundant computation and on-chip workspace size. A single annotated source code can be transformed by the CFDbuilder tool for good performance on a cache-based device, using minimal redundant computation, and alternatively for a GPU by specifying the necessary amount of redundant work.

We have applied this approach to the multifluid PPM algorithm specialized to the case of flow for which the Mach number is less than about 2 . Our automatic code translation tool transforms the special implementation 
of the mPPM algorithm, written in Fortran, in two stages to generate the output CUDA code. The performance report in Table 1 shows that the latest K80 GPU can achieve a performance of $432 \mathrm{Gflop} / \mathrm{s}$ and runs 1.7 times faster than 2 of the latest Intel Haswell CPUs. Specifying desired trade-offs between redundant computation and on-chip workspace size can enable the computational pipelining for high performance on GPUs. An enhanced CFDbuilder tool will assist the programmer to perform transformations for high performance on GPUs and relieve the programming burden in code translation to CUDA.

\section{Acknowledgements}

Development of PPM algorithms and codes, beginning with our work on the Los Alamos Roadrunner machine, that has led to the work reported here has been supported by contracts from the Los Alamos and Sandia National Laboratories. Our work at the University of Minnesota has also been supported by NSF through grant 1413548 and PRAC grant 1440025 for access to NCSA's Blue Waters system. We have also carried out tests on early examples of advanced hardware at Sandia's CSRI. This work was also performed under the auspices of the U.S. Department of Energy by Lawrence Livermore National Laboratory under Contract DE-AC52-07NA27344. LLNL-JRNL673849 .

\section{References}

[1] J. Michalakes, M. Vachharajani, GPU acceleration of numerical weather prediction, Parallel Processing Letters 18 (04) (2008) 531-548.

[2] J. Mielikainen, B. Huang, H.-L. A. Huang, M. D. Goldberg, Improved GPU/CUDA based parallel weather and research forecast (WRF) single moment 5-class (WSM5) cloud microphysics, Selected Topics in Applied Earth Observations and Remote Sensing, IEEE Journal of 5 (4) (2012) $1256-1265$.

[3] J. C. Linford, J. Michalakes, M. Vachharajani, A. Sandu, Automatic generation of multicore chemical kernels, Parallel and Distributed Systems, IEEE Transactions on 22 (1) (2011) 119-131. 
[4] T. Shimokawabe, T. Aoki, C. Muroi, J. Ishida, K. Kawano, T. Endo, A. Nukada, N. Maruyama, S. Matsuoka, An 80-fold speedup, 15.0 TFlops full GPU acceleration of non-hydrostatic weather model ASUCA production code, in: High Performance Computing, Networking, Storage and Analysis (SC), 2010 International Conference for, IEEE, 2010, pp. $1-11$.

[5] O. Fuhrer, C. Osuna, X. Lapillonne, T. Gysi, B. Cumming, M. Bianco, A. Arteaga, T. C. Schulthess, Towards a performance portable, architecture agnostic implementation strategy for weather and climate models, Supercomputing frontiers and innovations 1 (1).

[6] M. Bauer, S. Treichler, A. Aiken, Singe: leveraging warp specialization for high performance on GPUs, ACM SIGPLAN Notices 49 (8) (2014) 119-130.

[7] P.-H. Lin, J. Jayaraj, P. R. Woodward, A study of the performance of multifluid PPM gas dynamics on CPUs and GPUs, in: Application Accelerators in High-Performance Computing (SAAHPC), 2011 Symposium on, IEEE, 2011, pp. 42-51.

[8] P.-H. Lin, J. Jayaraj, P. Woodward, P.-C. Yew, A study of performance portability using piecewise-parabolic method (PPM) gas dynamics applications, Procedia Computer Science 9 (2012) 1988-1991.

[9] J. Jayaraj, P.-H. Lin, P. R. Woodward, P.-C. Yew, CFDbuilder: A library builder for computational fluid dynamics, in: Parallel \& Distributed Processing Symposium Workshops (IPDPSW), 2014 IEEE International, IEEE, 2014, pp. 1029-1038.

[10] P. R. Woodward, J. Jayaraj, R. Barrett, mPPM, viewed as a co-design effort, in: Proceedings of the 1st International Workshop on HardwareSoftware Co-Design for High Performance Computing, IEEE Press, 2014, pp. 33-40.

[11] P. R. Woodward, J. Jayaraj, P. Lin, G. Rockefeller, C. Fryer, G. Dimonte, W. Dai, R. Kares, Simulating Rayleigh-Taylor (RT) instability using PPM hydrodynamics at scale on roadrunner, Proc. NECDC (2010) $11-00061$. 
[12] P. R. Woodward, J. Jayaraj, P. Lin, M. Knox, C. L. Fryer, G. Dimonte, C. Joggerst, G. M. Rockefeller, W. Dai, R. J. Kares, et al., Simulating turbulent mixing from richtmyer-meshkov and rayleigh-taylor instabilities in converging geometries using moving cartesian grids, in: Proceedings of the 2012 Nuclear Explosives Code Development Conference (NECDC 2012), 2013.

[13] F. Herwig, P. R. Woodward, P.-H. Lin, M. Knox, C. Fryer, Global non-spherical oscillations in three-dimensional $4 \pi$ simulations of the hingestion flash, The Astrophysical Journal Letters 792 (1) (2014) L3.

[14] D. J. Quinlan, et al., ROSE compiler project, http://www. rosecompiler.org/.

[15] J. Stratton, N. Anssari, C. Rodrigues, I.-J. Sung, N. Obeid, L. Chang, G. D. Liu, W.-m. Hwu, et al., Optimization and architecture effects on GPU computing workload performance, in: Innovative Parallel Computing (InPar), 2012, IEEE, 2012, pp. 1-10.

[16] H.-S. Kim, I. El Hajj, J. Stratton, S. Lumetta, W.-M. Hwu, Localitycentric thread scheduling for bulk-synchronous programming models on CPU architectures, in: Proceedings of the 13th Annual IEEE/ACM International Symposium on Code Generation and Optimization, IEEE Computer Society, 2015, pp. 257-268.

[17] S. Unkule, C. Shaltz, A. Qasem, Automatic restructuring of GPU kernels for exploiting inter-thread data locality, in: Compiler Construction, Springer, 2012, pp. 21-40.

[18] A. Arteaga, D. Ruprecht, R. Krause, A stencil-based implementation of parareal in the $\mathrm{C}++$ domain specific embedded language STELLA, Applied Mathematics and Computation.

[19] T. Shimokawabe, T. Aoki, N. Onodera, High-productivity framework on GPU-rich supercomputers for operational weather prediction code ASUCA, in: Proceedings of the International Conference for High Performance Computing, Networking, Storage and Analysis, IEEE Press, 2014, pp. 251-261. 
[20] W. M. Hwu, Accelerating science with innovations in exascale computing, http://changes2014.csp.escience.cn/dct/page/70009, presentation at the CHANGES 2014 workshop, Beijing, China, Sept., 2014. 


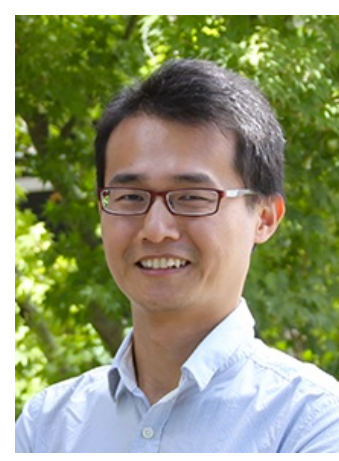

Dr. Pei-Hung Lin is a postdoctoral researcher in the Center for Applied Scientific Computing (CASC) at Lawrence Livermore National Laboratory. His research focus spans compiler optimization, parallel programming model, and domainspecific optimization. Dr. Lin received his Ph.D. degree in Computer Science from University of Minnesota in June 2013. He also holds M.S. in University of Minnesota and B.S. in National Tsing-Hua University, Taiwan.

Dr. Lin participated in several DOE and NSF research projects in large-scale scientific simulations. He has experiences with leading HPC systems including IBM Roadrunner system, Cray's XT5 (Kraken at NICS), Cray's XE6 \& XK7 (Blue Waters at NCSA) and IBM Blue Gene machines. He also actively participates in projects with innovative systems including the Nvidia's GPGPU and Intel's Xeon Phi. He was a core member in two demonstrations with Advanced Simulation and Computing (ASC) in Supercomputing conference 2009 and 2011.

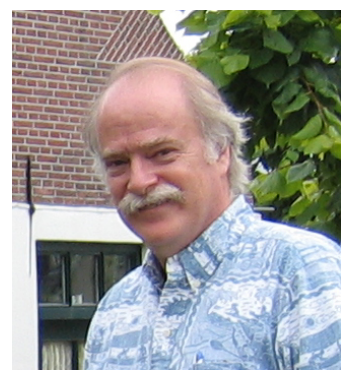

Dr. Paul R. Woodward is a professor of astronomy at the University of Minnesota, where he directs the Laboratory for Computational Science \& Engineering (LCSE). His research interests include computational fluid dynamics in astrophysics, especially in applications to stellar evolution theory where 3D effects play a major role. Woodward has a $\mathrm{PhD}$ in physics from the University of California, Berkeley. He is a member of the IEEE and SIAM. In 1999, he also received the Gordon Bell Prize in the performance category with several collaborators. 